\title{
Presentation of Gallbladder Chloroma in B-Mode Imaging and Contrast- Enhanced Ultrasound (CEUS) in a Patient with Acute Myelomonocytic Leukemia (AML M5)
}

\section{Introduction}

Chloromas (myeloid sarcoma) are solid tumors consisting of primitive myeloid precursors and have been described in acute myeloid leukemia (AML) and myeloproliferative disorders (Aznab, Mozaffar; Kamalian, Naser; Beiki, Omid et al., Int J Hematol Oncol Stem Cell Res 2015; 9 (1), S. 50-54). In AML, they are observed in 3-8\% of all cases, while the frequency varies (J. C.; Edenfield, W. J.; Shields, D. J. et al., Extramedullary myeloid cell tumors in acute nonlymphocytic leukemia: a clinical review. In: J Clin Oncol 1995, 13 (7), S. 1800-1816). Chloromas can be observed at diagnosis, precede the diagnosis, occur during the course of the disease or manifest as a relapse of the AML (Aznab, Mozaffar; Kamalian, Naser; Beiki, Omid et al., Int J Hematol Oncol Stem Cell Res 2015; 9 (1), S. 50-54). They can affect every organ system, with infiltration of bone, skin, soft tissues and lymph nodes being observed most frequently (Aznab, Mozaffar; Kamalian, Naser; Beiki, Omid et al., Int J Hematol Oncol Stem Cell Res 2015; 9 (1), S. 50-54), J. C.; Edenfield, W. J.; Shields, D. J. et al., Extramedullary myeloid cell tumors in acute nonlymphocytic leukemia: a clinical review. In: J Clin Oncol 1995, 13 (7), S. 1800-1816). Single case reports describe chloromas of the gallbladder and the bile ducts (Azin, Arash; Racz, Jennifer M.; Carolina Jimenez, M. et al. Int J Surg Case Rep 2014;5 (6), S. 302-305). In summary, we present the first description of contrast-enhanced ultrasound (CEUS) patterns of a histologically confirmed gallbladder chloroma.

\section{History and Clinical Findings}

A 64-year-old patient with acute myelomonocytic leukemia (AML M5) was hospitalized for consolidation therapy. The patient achieved complete remission after standard induction therapy. No excess of blasts was detected in histological examinations of the bone marrow and the patient showed no clinical signs of extramedullary AML. From day 20 after therapy initiation, laboratory testing indicated an acute infection with concomitant increasing serum bilirubin levels and a cholestatic liver enzyme pattern (max. GOT: $105 \mathrm{U} / \mathrm{I}$, max. GPT: 172 U/l, max. alkaline phosphatase $428 \mathrm{U} / \mathrm{I}$ ). Platelet counts ranged between 20 and $60 \mathrm{G} / \mathrm{l}$ and the leukocytes remained less than $1 \mathrm{G} / \mathrm{l}$. The patient received transfusion of erythrocyte concentrates without signs of bleeding (hemoglobin levels ranged from 7 to $10 \mathrm{G} / \mathrm{l})$,

\section{Examination}

The abdominal ultrasound showed an intraand extrahepatic cholestasis ( $\mathbf{F i g}$. 1a). B-mode imaging revealed an enlarged, wall-accented gallbladder with intraluminal echogenic sludge and small nodules in the wall. For further differentiation contrast-enhanced ultrasound (CEUS) was performed. Therefore, $2.4 \mathrm{ml}$ of the contrast media SonoVue were applied and rinsed with $10 \mathrm{ml}$ of $\mathrm{NaCl}$. CEUS of the nodules ( $\triangleright$ Fig. 1b-d) and the intraluminal sludge showed enhancement, indicating vital tissue, and
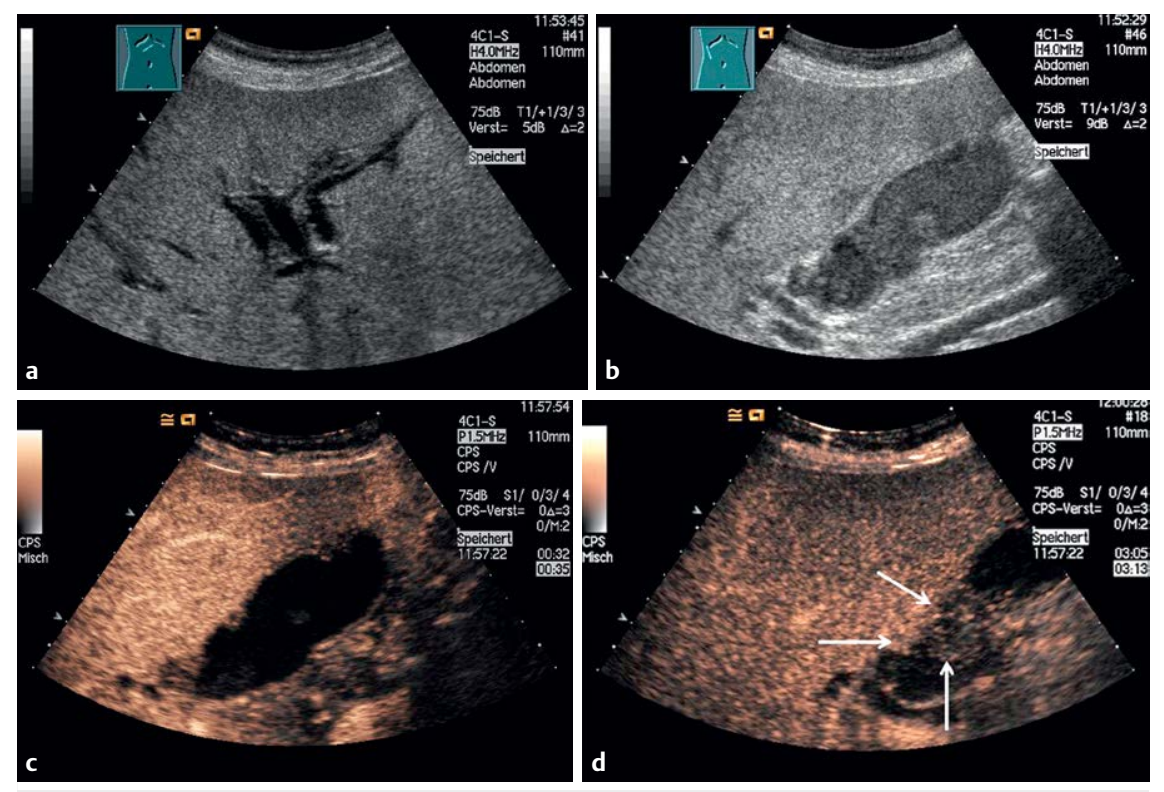

- Fig. 1 a Intrahepatic cholestasis. b Thickening of the gallbladderwall with parietal noduli and intraluminal enhancement in a patient with acute myelomonocytic leucemia. c Enhancement of the parietal noduli in the gallbladderwall in the arterial phase of CEUS. $\mathbf{d}$ Enhancement of the parietal noduli in the gallbladderwall and the intraluminal material in CEUS. 


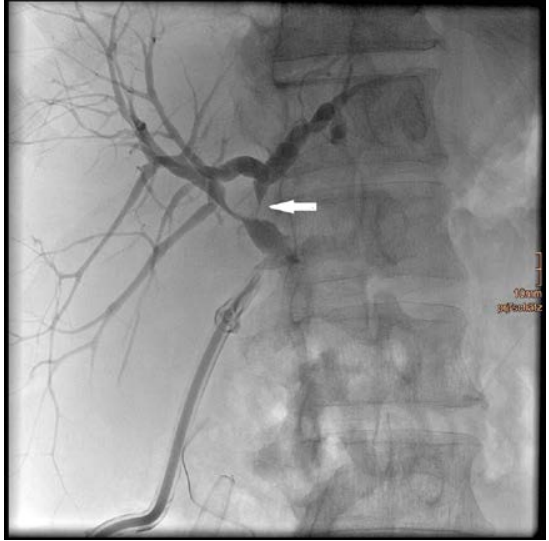

- Fig. 2 Postoperative radiological control of the percutaneous biliary drainage with evidence of a stenosis in the area of the bifurcation of the bile duct.

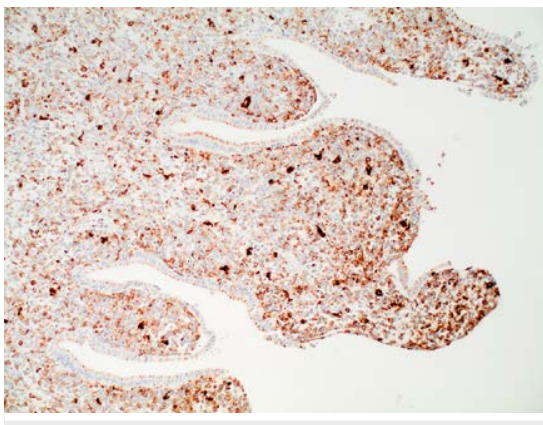

- Fig. 3 Gallbladderwall with infiltration of AML, immunohistochemistry CD 68 (marker FAB M5).

the inserted drainage tube ( $\vee$ Fig. 2$)$. Histopathological examination showed a tumorous infiltration of the gallbladder wall and the cystic duct with AML M5 blasts ( Fig. 3).

\section{Discussion}

The main differential diagnoses of marked echogenic material of the gallbladder wall and gallbladder lumen in B-mode imaging are gallbladder carcinoma, gallstones, gallbladder polyps, sludge, and special forms of chronic cholecystitis. In 2011, the EFSUMB first recommended the application of contrast-enhanced ultrasound (CEUS) outside the liver (Piscaglia, F.; Nolsoe, C.; Dietrich, C. F. et al., Ultraschall in Med 2012, 33 (1), S. 33-59). CEUS may be helpful in the diagnosis of pathologic processes of the gallbladder especially in the discrimination of vascular from avascular tissue (Piscaglia, F.; Nolsoe, C.; Dietrich, C. F. et al., Ultraschall in Med 2012, 33 (1), S. 33-59). Gallstones or sludge as non-vascularized waste products can be differentiated from vascularized processes that indicate growth of a vital tumor (Piscaglia, F.; Nolsoe, C.; Dietrich, C. F. et al., Ultraschall in Med 2012, 33 (1), S. 33-59). XU et al. observed that the application of CEUS is helpful to assess gallbladder wall thickening of unclear malignancy (Xu, Jun-Mei; Guo, Le-Hang; Xu, HuiXiong et al. Ultrasound Med Biol 2014, 40 (12), S. 2794-2804). Malignant gallbladder wall thickening was associated with intralesional vessels, inhomogeneous enhancement of the gallbladder wall, rapid flushing of the contrast media and discontinuous wall stratification (Xu, Jun-Mei; Guo, LeHang; Xu, Hui-Xiong et al. Ultrasound Med Biol 2014, 40 (12), S. 2794-2804). Of course in cases of pathologic enhancement of the gallbladder wall or lumen, histologic confirmation is warranted. In our case, B-mode imaging showed gallbladder wall thickening with parietal noduli and intraluminal masses, morphologically resembling sludge. Both findings indicate several malignant and non-malignant differential diagnoses. Inhomogeneous enhancement on CEUS strongly indicated a malignant process which was later histologically confirmed as a chloroma. Chloromas, extramedullary tumorous hematopoiesis and hematoma of the gallbladder or of bile ducts are a possible differential diagnosis in patients with acute or chronic myeloproliferative diseases and unexplained cholestasis or inflammatory processes of the biliary tract. CEUS can be a valuable tool for the diagnostic workup.

\section{Conflict of Interest}

The authors have no conflict of interest to disclose.

\section{Authors}

Corinna Trenker ${ }^{1}$, Marius Dohse ${ }^{2}$,

Stephan K. Metzelder ${ }^{1}$, Anette Ramaswamy ${ }^{3}$, Walter Hundt ${ }^{3}$, Christian Görg ${ }^{4}$

\section{Affiliations}

1 University hospital Gießen and Marburg, Department of Haematology, Oncology and Immunology, Philipps University Marburg, Marburg, Germany

2 Universitäsklinikum Gießen und Marburg, Standort Marburg, Department of Pathology, Philipps-University Marburg, Marburg, Germany

3 Universitatsklinikum Giessen und Marburg - Standort Marburg, Dianostische Und Interventionelle Radiologie, Marburg, Germany

4 University hospital Gießen and Marburg, Interdiciplinary ultrasound center

\section{Correspondence}

Dr. Corinna TrenkerUKGM

Klinik für Innere Medizin, Schwerpunkt Hämatologie

Onkologie und Immunologie

Universitätsklinikum Giessen und Marburg,

Standort Marburg

Baldingerstraße

35043, Marburg

Germany

+49/6421/5862 599

trenker@med.uni-marburg.de

\section{Bibliography}

DOI https://doi.org/10.1055/s-0043-118527 Ultrasound Int Open 2017; 3: E163-E164 (c) Georg Thieme Verlag KG Stuttgart . New York

ISSN 2199-7152

\section{(우(1) $(2) \ominus$}

